\title{
A Model for Harmonics on Stringed Instruments
}

\author{
A. BAMBerger, J. RAUCH \& M. TAYLOR
}

Communicated by J. SERRIN

\section{Introduction}

We propose a model to explain the playing of "harmonics" on stringed instruments. The interesting phenomenon here is that placing a finger lightly at one of the nodes of the low frequency harmonics seems to force the string to play a note that sounds like a superposition of those normal modes with nodes at the location of the finger. For example, if the finger is placed $1 / 4$ the way down the string the note that is heard is two octaves above the fundamental (Figure 1).

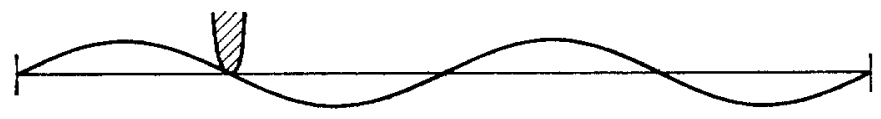

Fig. 1

Pressing hard at that place on the string would yield a note with fundamental much lower (see Figure 2). With care one can play harmonics 4 or 5 octaves above the fundamental. It is very striking for example to hear a fat bass string play these shrill high tones vibrating along their entire length. A second aspect is that if the finger is placed at a point which is not a node of a low frequency normal node, the observed sound is a rapidly dying thud.

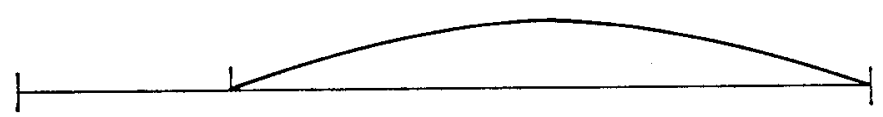

Fig. 2

The problem we pose is to construct a model for the lightly placed finger which explains these observations. It turns out that a strong frictional resistance which is localized in a very small region has the desired properties. More precisely, 
the model we propose for the string occupying the interval $0 \leqq x \leqq \pi$ and fixed at the endpoints $x=0$ and $\pi$ is

$$
\begin{gathered}
u_{t t}+b(x) u_{t}=u_{x x} ; \quad 0 \leqq x \leqq \pi, \quad t \geqq 0, \\
u(t, 0)=u(t, \pi)=0 ; \quad t \geqq 0 .
\end{gathered}
$$

Here the frictional resistance $b(x)$, which models the finger pressure, is assumed to be positive and strongly localized near a point $a \in(0, \pi)$, as illustrated in Figure 3. Existence, uniqueness, and qualitative behavior of solutions of the problem are developed largely from the law of energy decay

$$
\frac{d}{d t} \int_{0}^{\pi}\left(u_{t}^{2}+u_{x}^{2}\right) d x=-2 \int_{0}^{\pi} b(x) u_{t}^{2}(x) d x \leqq 0 .
$$

The analysis proceeds in two steps. First, we show that for highly localized $b$, the behavior of (1.1) is approximated by that of the singular equation

$$
u_{t t}+\alpha \delta(x-a) u_{t}=u_{x x}, \quad \alpha=\int_{0}^{\pi} b(x) d x .
$$

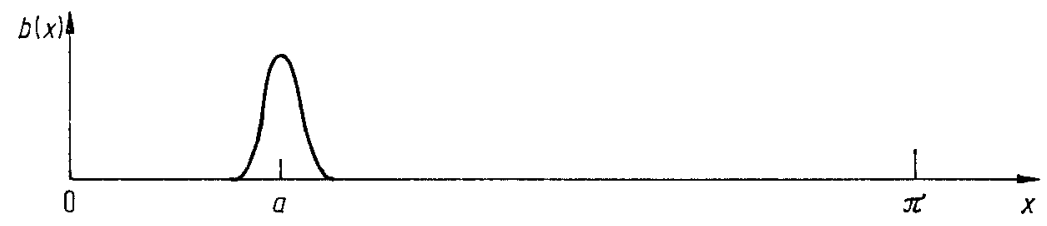

Fig. 3

This formally reasonable limit is equivalent to the wave equation for $x \neq a$ supplemented by a transmission condition at $x=a$. The second step is a fairly precise analysis of this limiting equation. Among other things, we show that if $a / \pi$ is irrational, then all solutions tend to zero as $t \rightarrow \infty$, while if $a / \pi$ is rational, the components of $u$ in the span of the modes which vanish at $x=a$ propagate as if there were no friction while the components orthogonal (in the natural scalar product given by the energy) to these decay exponentially. These results mirror the observed phenomena described above.

The appropriateness of our model and a possible experimental test are discussed in $\S 4$, after the precise results are in hand.

\section{The limiting transmission problem}

To study the behavior of (1.1), (1.2) with highly localized friction, we investigate the limiting behavior as $b(x)$ becomes more and more localized. It turns out that the limiting behavior corresponds quite closely to the above observations concerning stringed instruments. In this section, we will show that the limiting behavior is given by solving a specific transmission problem, and an analysis of this problem is given in the next section. 
First, we cast the basic problem in the framework of the theory of semigroups of operators. Consider the pair $U \equiv\left(u_{t}, u\right)$ as an element of the Hilbert space $\mathscr{H}=L_{2}[0, \pi] \oplus \stackrel{0}{H}_{1}[0, \pi]$, where $\stackrel{0}{H}_{1}[0, \pi]$ is the completion of $C_{0}^{\infty}(0, \pi)$ in the norm $\left(\int_{0}^{\pi} u^{2}+u_{x}^{2} d x\right)^{\frac{1}{2}}$. The mixed problem (1.1)-(1.2) is equivalent to the evolution equation

$$
U_{t}=\Gamma U
$$

where

$$
\begin{gathered}
\Gamma=\left(\begin{array}{cc}
-b & D^{2} \\
I & 0
\end{array}\right), \quad D=\frac{\partial}{\partial x} \\
\mathscr{D}(\Gamma)=\stackrel{0}{H_{1}}[0, \pi] \oplus\left(H_{2}[0, \pi] \cap \stackrel{0}{H}_{1}[0, \pi]\right) .
\end{gathered}
$$

It is a simple matter, using the theory of ordinary differential equations, to show that $\Gamma$ so defined is a maximal dissipative operator. We will present a similar but slightly harder proof for the operator $G_{\alpha}$ which occurs further on, and therefore omit the details of the present argument. The theory of semigroups provides a solution of the differential equation (2.1) with initial condition

$$
U(0)=(g, f) \in \mathscr{H},
$$

the solution $U$ being a continuous function of $t$ with values in $\mathscr{H}$. In addition, if $(g, f) \in \mathscr{D}\left(\Gamma^{3}\right)$, it is not hard to show that the associated function $u(t, x)$ is a classical solution of the mixed problem (1.1)-(1.2), with $u(0, x)=f, u_{t}(0, x)=g$. There are, of course, other ways to treat this mixed problem, using for example the method of characteristics [1, chap. 5], or the theory of symmetric positive systems $[2,8]$. We have chosen the present approach because it seems to yield the strongest results when we consider the limiting behavior as $b$ becomes more localized.

For future use, we record one more fact about $\Gamma$. The equation $(I-\Gamma) U=F$ for $U=(v, w)$ and $F=\left(F_{1}, F_{2}\right)$ in $\mathscr{H}$ is equivalent to the following equations:

$$
w-v=F_{2}
$$

and

$$
\int_{0}^{\pi}\left(w \phi+b v \phi+w_{x} \phi_{x}\right) d x=\int_{0}^{\pi} F_{1} \phi d x, \quad \text { for all } \forall \phi \in \stackrel{0}{H_{1}}[0, \pi] .
$$

This weak formulation is ideally suited to our needs. We consider a sequence of nonegative friction coefficients $b_{n} \in C^{\infty}[0, \pi]$ with the property that

$$
\lim _{n} \int_{0}^{\pi} b_{n}(x) \psi(x) d x=\alpha \psi(a), \quad \text { for all } \forall \psi \in C[0, \pi],
$$

where $0 \leqq \alpha<\infty$. If $b_{n}$ satisfies (2.5) and $u_{n}$ is the solution of the mixed problem (1.1)-(1.2) with initial data $(g, f)$, then as $n \rightarrow \infty$ the functions $u_{n}$ converge 
to the solution $u$ of the transmission problem

$$
\begin{gathered}
u_{t t}-u_{x x}=0 \quad \text { for } x \in[0, \pi] \backslash a, \quad t \geqq 0, \\
{\left[u_{t}\right]=0 \text { and } \alpha u_{t}=\left[u_{x}\right] \quad \text { at } x=a, \quad t \geqq 0,} \\
u=0 \quad \text { at } x=0, \pi,
\end{gathered}
$$

with $\left(u_{t}(0, x), u(0, x)\right)=(g, f)$. The quantity $[h]$ at $x=a$ is the jump $h(a+0)$ $-h(a-0)$ in $h$ at the point $a$.

There is a semigroup formulation of this problem also. For the state vector $U=\left(u_{t}, u\right) \in \mathscr{H}$ we get the equation

where

$$
U_{t}=G_{\alpha} U
$$

$$
G_{\alpha}=\left(\begin{array}{ll}
0 & D^{2} \\
I & 0
\end{array}\right) \text { for } x \neq a
$$

and

$$
\mathscr{D}\left(G_{\alpha}\right)=\left\{(v, w) \in \stackrel{0}{H_{1}}[0, \pi] \oplus\left(H_{2}[0, a] \cap H_{2}[a, \pi]\right) \mid \alpha v=\left[w_{x}\right] \text { at } x=a\right\} .
$$

This is reasonable, since formally $\Gamma$ approaches $\left(\begin{array}{cc}-\alpha \delta_{a} & D^{2} \\ I & 0\end{array}\right)$ while the equation $D^{2} w-\alpha \delta_{a} v=F_{1}$ translates to $D^{2} w=F_{1}$ for $x \neq a$ and $\alpha v=\left[w_{x}\right]$ at $x=a$.

We show that $G_{\alpha}$ as defined above is a maximal dissipative operator. First, for all $U=(v, w) \in \mathscr{D}\left(G_{\alpha}\right)$ we have

$$
\mathscr{R} e(G U, U)_{\mathscr{K}}=-\alpha v^{2}(a) \leqq 0,
$$

so $G_{\alpha}$ is dissipative. To see that $\mathscr{R} g\left(I-G_{\alpha}\right)=\mathscr{H}$ suppose that $F=\left(F_{1}, F_{2}\right) \in \mathscr{H}$; then the equation $\left(I-G_{\alpha}\right) U=F$ is equivalent to

$$
\begin{aligned}
& (v, w) \in \mathscr{D}(G), \quad w-v=F_{2}, \\
& v-D^{2} w=F_{1} \text { on }[0, \pi] \backslash a .
\end{aligned}
$$

Using the first equation to eliminate $v$ from the second, we get

$$
-D^{2} w+w=F_{1}-F_{2}, \quad x \neq a .
$$

There is a two parameter family of solutions of this equation which, in addition, satisfy the conditions $w=0$ at $x=0, \pi$; in fact, we may take $w^{\prime}(0), w^{\prime}(\pi)$ as the parameters. In addition to (2.9) $w$ must satisfy

$$
[w]=0 \text { and } \alpha w-\left[w_{x}\right]=F_{2} \text { at } x=a,
$$

where the second condition comes from eliminating $v$ from the transmission condition.

To see that these restrictions uniquely determine $w^{\prime}(0), w^{\prime}(\pi)$ we need only show that the map

$$
\left(w^{\prime}(0), w^{\prime}(\pi)\right) \rightarrow\left([w], \alpha w(a+)-\left[w_{x}\right]_{a}\right)
$$


is a nonsingular linear transformation from $\mathbb{R}^{2}$ into itself. For this, it suffices to show that the map is injective. If $\left(w^{\prime}(0), w^{\prime}(\pi)\right) \rightarrow(0,0)$, then the associated function $w$ satisfies

$$
\begin{gathered}
\left(I-D^{2}\right) w=0 \text { for } x \neq a, \\
{[w]=\alpha w-\left[w_{x}\right]=0 \text { at } x=a,} \\
w=0 \text { at } x=0, \pi .
\end{gathered}
$$

Integrating by parts in the identity

then yields

$$
\int_{0}^{a} w\left(I-D^{2}\right) w d x+\int_{a}^{\pi} w\left(I-D^{2}\right) w d x=0
$$

$$
\int_{0}^{\pi}\left((D w)^{2}+w^{2}\right) d x+\alpha w^{2}(a)=0
$$

so $w=0$. Thus $w$ is uniquely determined by (2.9), (2.10). Setting $v=w-F_{2}$ the pair $U=(v, w)$ satisfies $\left(I-G_{\alpha}\right) U=F$ and proof of maximality is complete.

The next theorem asserts the convergence of the solutions of the mixed problem (1.1)-(1.2), with friction coefficients, $b_{n}$ satisfying (2.5), to the solution of the above transmission problem.

Theorem 1. If the nonnegative friction coefficients $b_{n}$ satisfy (2.5), and if $\Gamma_{n}$ are the associated maximal dissipative operators, then for each $t \geqq 0$

$$
s-\lim _{n \rightarrow \infty} e^{t \Gamma_{n}}=e^{t G_{x}}
$$

and the convergence is uniform on compact time intervals.

Proof. We apply the Trotter-Kato theorem [4, chapter 9, theorem 2.16], thereby reducing the problem to showing that for every $F \in \mathscr{H}$

$$
\left(I-\Gamma_{n}\right)^{-1} F \rightarrow\left(I-G_{\alpha}\right)^{-1} F \text { in } \mathscr{H} \text {. }
$$

Let $U_{n}=\left(v_{n}, w_{n}\right) \equiv\left(I-\Gamma_{n}\right)^{-1} F$. We first show that $U_{n}$ converges weakly in $\mathscr{H}$ to $\left(I-G_{\alpha}\right)^{-1} F$. Notice that

$$
\left\|U_{n}\right\|_{\mathscr{H}} \leqq\left\|\left(I-\Gamma_{n}\right)^{-1}\right\|\|F\|_{\mathscr{H}} \leqq\|F\|,
$$

so $\left\{U_{n}\right\}$ is weakly compact in $\mathscr{H}$. Let $U=(v, w)$ be a weak limit point and choose a subsequence $U_{n_{k}}$ converging weakly to $U$ (symbolically, $U_{n_{k}} \rightarrow U$ ). We must show that $U=\left(I-G_{\alpha}\right)^{-1} F$.

From equation (2.3), we find $w_{n_{k}}-v_{n_{k}}=F_{2}$. Hence, passing to the limit $k \rightarrow \infty$ we have $w-v=F_{2}$.

Similarly, equation (2.4) holds for $w_{n_{k}}, v_{n_{k}}$ provided $b$ is replaced by $b_{n_{k}}$. Passing to the limit, we obtain

$$
\int_{0}^{\pi}(w \phi+D w D \phi) d x+\lim \int b_{n_{k}} v_{n_{k}} \phi d x=\int_{0}^{\pi} F_{1} \phi d x, \quad \forall \phi \in \stackrel{0}{H_{1}}[0, \pi] .
$$


By (2.5), if we consider $b_{n} \phi$ as an element of $(C[0, \pi])^{\prime}$, the sequence $b_{n} \phi$ converges weakly to $\alpha \phi(a) \delta_{a}$. However $v_{n_{k}}=w_{n_{k}}-F_{2}$ converges weakly to $v$ in $\stackrel{0}{H}_{1}[0, \pi]$ and therefore uniformly. Thus the limit above is $\alpha v(a) \phi(a)$, and we have

$$
\int_{0}^{\pi}(w \phi+D w D \phi) d x+\alpha v(a) \phi(a)=\int_{0}^{\pi} F_{1} \phi d x,
$$

for all $\phi \in \stackrel{0}{H}_{1}[0, \pi]$. With the help of the identity $w-v=F_{2}$, the condition (2.11) is easily shown to be equivalent to the equation $\left(I-G_{\alpha}\right) U=F$. This proves the weak convergence $U_{n} \rightarrow U$.

To prove the strong convergence, we investigate the sequence $\left\|U_{n}\right\|_{\mathscr{H}}$. To do this, set $\phi=w_{n}$ in (2.4) to obtain

$$
\int_{0}^{\pi}\left(w_{n}^{2}+\left(D w_{n}\right)^{2}\right) d x=\int_{0}^{\pi} F_{1} w_{n} d x-\int_{0}^{\pi} b_{n} v_{n} w_{n} d x
$$

The limit of the right hand side as $n \rightarrow \infty$ is

$$
\int F_{1} w d x-\alpha w(a) v(a)
$$

which is precisely $\int_{0}^{\pi} w^{2}+(D w)^{2} d x$ as can be seen by choosing $\phi=w$ in (2.11). This takes care of the $w$ component; that is, $w_{n} \rightarrow w$ in $\stackrel{0}{H_{1}}[0, \pi]$. It follows then that $v_{n}=w_{n}-F_{2} \rightarrow v$ in $L_{2}[0, \pi]$, and the proof is complete.

If one considers $b_{n}(x)$ with $b_{n} \geqq 0$, this theorem shows that $\int b_{n}(x) d x$ is an appropriate measure of the strength of the frictional force, since if $\int b_{n}(x) d x$ $\rightarrow \alpha \geqq 0$, the limiting behavior is given by (2.6)-(2.8). Next, we turn our attention to the case of extremely large friction. Suppose $b_{n} \geqq 0$, and

$$
\begin{gathered}
\int b_{n}(x) d x=\beta_{n} \nearrow \infty \text { as } n \rightarrow \infty, \\
\limsup _{\substack{x \in K \\
n \in Z_{+}}} b_{n}(x)=0, \text { for all compact } K \subset[0, \pi] \backslash a .
\end{gathered}
$$

The second hypothesis asserts that, away from the point $a$, the functions $b_{n}$ are bounded uniformly, a hypothesis expressing the localization of the $b_{n}$ near $a$.

Suppose that $U_{n}$ is the solution of the wave equation (1.1)-(1.2) with Cauchy data $\frac{\partial u_{n}}{\partial t}(0)=\psi, u_{n}(0)=\varphi$ and energy at time $t$ given by

$$
\left\|U_{n}(t)\right\|_{\mathscr{H}}^{2} \equiv \int_{0}^{\pi}\left(u_{t}^{2}(t, x)+u_{x}^{2}(t, x)\right) d x .
$$

The formula for energy decay is

In particular, for all $n$,

$$
\left\|U_{n}(T)\right\|_{\mathscr{H}}^{2}=\left\|U_{n}(0)\right\|_{\mathscr{H}}^{2}-\int_{0}^{T} \int_{0}^{\pi} b_{n}\left(\frac{\partial u_{n}}{\partial t}\right)^{2} d x d t
$$

$$
\int_{0}^{T} \int_{0}^{\pi} b_{n}\left(\frac{\partial u_{n}}{\partial t}(t, x)\right)^{2} d x d t \leqq \int_{0}^{\pi}\left(\psi(x)^{2}+\varphi^{\prime}(x)^{2}\right) d x .
$$


This implies that if we consider $\frac{\partial u_{n}}{\partial t}(., x) \in L^{2}[0, T]$ for each $x$, the weighted averages satisfy

$$
\frac{1}{\beta_{n}} \int_{0}^{\pi} b_{n}(x)\left\|\frac{\partial u_{n}}{\partial t}(., x)\right\|_{L^{2}[0, T]}^{2} d x \rightarrow 0 \text { as } n \rightarrow \infty .
$$

Now $\left\{U_{n}\right\}$ is bounded in $H^{1}((0, T) \times(0, \pi))$, and

$$
H^{1}((0, T) \times(0, \pi)) \rightarrow C\left([0, \pi] ; L^{2}(0, T)\right) \text { compactly; }
$$

thus $\left\{\frac{\partial u_{n}}{\partial t}\right\}$ lies in a compact subset of $C\left([0, \pi] ; H^{-1}(0, T)\right)$. Since equation (2.13) implies that

it follows that

$$
\frac{1}{\beta_{n}} \int_{0}^{\pi} b_{n}(x)\left\|\frac{\partial u_{n}}{\partial t}(., x)\right\|_{H^{-1}(0, T)}^{2} d x \rightarrow 0 \text { as } n \rightarrow \infty
$$

$$
\frac{\partial u_{n}}{\partial t}(., a) \rightarrow 0 \text { in } H^{-1}(0, T), \quad \text { as } n \rightarrow \infty
$$

It is therefore reasonable to expect that $u_{n}$ tends in the limit to the unique solution $u \in H_{1}((0, T) \times(0, \pi))$ of the mixed problem

$$
\begin{gathered}
u_{t t}-u_{x x}=0 \text { in }(0, T) \times((0, \pi) \backslash a), \\
u(\cdot, 0)=u(\cdot, \pi)=0 \text { on }(0, T), \\
\frac{\partial u}{\partial t}(., a)=0 \text { on }(0, T), \\
u(0, .)=\varphi \text { on }(0, \pi), \\
\frac{\partial u}{\partial t}(0, .)=\psi \text { on }(0, \pi) .
\end{gathered}
$$

Note that this limiting problem is energy conserving.

Theorem 1'. If (2.12) holds and the Cauchy data $(\psi, \varphi)$ is in $\mathscr{H}$, then for each $t \geqq 0$

$$
\left(\frac{\partial u_{n}}{\partial t}(t), u_{n}(t)\right) \rightarrow\left(\frac{\partial u}{\partial t}(t), u(t)\right) \text { in } \mathscr{H} \text { as } n \rightarrow \infty,
$$

where $u \in C([0, T]: \mathscr{H})$ is the solution of the mixed problem $(2.15)-(2.19)$.

Proof. Because the energy is a decreasing function of time for each $U_{n}$, it suffices to prove the theorem for a set of Cauchy data which is dense in $\mathscr{H}$. Thus, we may assume there is an $\eta>0$ and a $C \in \mathbb{R}$ such that

and

$$
\psi=0 \text { on }[a-\eta, a+\eta]
$$

$$
\varphi=C \text { on }[a-\eta, a+\eta]
$$


It follows from the finite speed of propagation that, for all $n$,

$$
u_{n}=C \text { on }\{(t, x):|t|+|x-a|<\eta\} .
$$

We begin by showing that $u_{n} \rightarrow u$ weakly in $H^{1}((0, T) \times(0, \pi))$. To do this, we show that every subsequence of the $b_{n}$ has a further subsequence converging weakly to $u$. The crucial observations are

$$
\left\{u_{n}\right\} \text { is bounded in } C\left([0, T]: \stackrel{0}{H^{1}}(0, \pi)\right)
$$

and

$$
\left\{\frac{\partial u_{n}}{\partial t}\right\} \text { is bounded in } C\left([0, T]: L^{2}(0, \pi)\right),
$$

both consequences of energy decay. From the differential equation (1.1) and condition $(2.12)_{\mathrm{ii}}$, we see that, for any compact interval $K$ in $[0, \pi] \backslash a$,

$$
\left\{\frac{\partial^{2} u_{n}}{\partial t^{2}}\right\} \text { is bounded in } C\left([0, T]: H^{-1}(K)\right) \text {. }
$$

It follows that, given any subsequence of the $u_{n}$, we may choose a further subsequence $u_{n_{j}}$ such that in the weak star topologies

$$
\begin{gathered}
u_{n_{j}} \rightarrow w \text { in } L^{\infty}\left((0, T): \stackrel{0}{H^{1}}(0, \pi)\right), \\
\frac{\partial u_{n_{j}}}{\partial t} \rightarrow \frac{\partial w}{\partial t} \text { in } L^{\infty}\left((0, T): L^{2}(0, \pi)\right), \\
\frac{\partial^{2} u_{n_{j}}}{\partial t^{2}} \rightarrow \frac{\partial^{2} w}{\partial t^{2}} \text { in } L^{\infty}\left((0, T): H_{\mathrm{loc}}^{-1}((0, \pi) \backslash a)\right) .
\end{gathered}
$$

It follows immediately that

$$
\begin{gathered}
w \in H^{1}((0, T) \times(0, \pi)), \\
w_{t t}-w_{x x}=0 \text { in }(0, T) \times((0, \pi) \backslash a), \\
w=0 \text { in }(0, T) \times\{0\} \text { and }(0, T) \times\{\pi\}, \\
w=\phi \text { on }\{0\} \times(0, \pi) .
\end{gathered}
$$

From (2.14) we see that, as an element of $H^{-1}(0, T)$,

$$
\frac{\partial w}{\partial t}=0 \text { on }(0, T) \times\{a\}
$$

From (2.20) we get $\frac{\partial w}{\partial t}=0$ on $(0, \eta) \times\{a\}$, and therefore

$$
\frac{\partial w}{\partial t}=0 \text { on }[0, T) \times\{a\}
$$


We must show that at $t=0, \frac{\partial w}{\partial t}=\psi$. From (2.23)-(2.35), it follows that

$$
\left.\left.\frac{\partial u_{n_{j}}}{\partial t}\right|_{t=0} \rightarrow \frac{\partial w}{\partial t}\right|_{t=0} \text { weakly in } L_{\mathrm{loc}}^{2}((0, \pi) \backslash a),
$$

so that

$$
\left.\frac{\partial w}{\partial t}\right|_{t=0}=\psi \text { on }(0, \pi) \backslash a
$$

However, from (2.21),

$$
\psi=\frac{\partial w}{\partial t}=0 \text { on }\{0\} \times(a-\eta, a+\eta),
$$

which together with (2.27) yields the desired result.

We have proved that $u_{n} \rightarrow u$ weakly in $H^{1}((0, T) \times(0, \pi))$. The functions $u_{n}$ and $u$ lie in the closed subspace of $\mathscr{H}$ consisting of functions vanishing at $(0, T) \times\{0\}$. On this subspace the quantity

$$
\|u\|_{\varepsilon}^{2} \equiv \int_{0}^{T} \int_{0}^{\pi}\left(u_{t}^{2}+u_{x}^{2}\right) d x d t
$$

furnishes a norm equivalent to that of $H^{1}((0, T) \times(0, \pi))$. Clearly

$$
\left\|u_{n}\right\|_{\varepsilon}^{2} \leqq T\|(\psi, \varphi)\|_{\mathscr{H}}^{2}=\|u\|_{\varepsilon}^{2} .
$$

This norm inequality together with weak convergence implies that $u_{n} \rightarrow u$ strongly in $H^{1}((0, T) \times(0, \pi))$.

It remains to show that, for each $t \in[0, T]$,

$$
\left(\frac{\partial u_{n}}{\partial t}(t), u_{n}(t)\right) \rightarrow\left(\frac{\partial u}{\partial t}(t), u(t)\right) \text { in } \mathscr{H} .
$$

From the laws of energy decrease, we know that $\left\{u_{n}(t)\right\}$ is bounded in $H^{1}(0, \pi)$ and from the weak convergence in $H^{1}((0, T) \times(0, \pi))$ we know that $u_{n}(t) \rightarrow u(t)$ in $L^{2}(0, \pi)$. It follows that $u_{n}(t) \rightarrow u(t)$ weakly in $H^{1}(0, \pi)$. Similarly, $\left\{\frac{\partial u_{n}}{\partial t}(t)\right\}$ is bounded in $L^{2}(0, \pi)$, and (2.23) and (2.25) imply that

$$
\left.\frac{\partial u_{n}}{\partial t}(t) \rightarrow \frac{\partial u}{\partial t}(t) \text { weakly in } L_{\mathrm{loc}}^{2}((0, \pi) \backslash a)\right) \text {. }
$$

Consequently

$$
\frac{\partial u_{n}}{\partial t}(t) \rightarrow \frac{\partial u}{\partial t}(t) \text { weakly in } L^{2}(0, \pi)
$$

Thus

$$
\left(\frac{\partial u_{n}}{\partial t}(t), u_{n}(t)\right)-\left(\frac{\partial u}{\partial t}(t), u(t)\right) \text { weakly in } \mathscr{H}
$$


In addition,

$$
\left\|\left(\frac{\partial u_{n}}{\partial t}(t), u_{n}(t)\right)\right\|_{\mathscr{H}} \leqq\left\|(\psi, \varphi)_{\mathscr{H}}\right\|=\left\|\left(\frac{\partial u}{\partial t}(t), u(t)\right)\right\|_{\mathscr{H}},
$$

and the strong convergence in $\mathscr{H}$ follows.

\section{Analysis of the transmission problems}

In this section, we make a qualitative analysis of the transmission problems associated with localized friction. A crucial role is played by the position $a$ of the friction and in particular the rationality or irrationality of $a / \pi$. The main results describe the spectrum of $G_{\alpha}$ and the asymptotic behavior of $e^{z G_{\alpha}}$ as $t \rightarrow \infty$. First, we present some results which do not depend on the value of $a$.

Theorem 2. The family $G_{\alpha}$ is holomorphic in $\alpha$ for all $\alpha \in \mathbb{C}$. The operators $G_{\alpha}$ have compact resolvents and the eigenvalues of $G_{\alpha}$ are simple, that is, have multiplicity one. The eigenvalues and eigenprojections are analytic functions of a for $0<a<\pi$. For $\alpha \geqq 0$, the spectrum of $G_{\alpha}$ is contained in the half plane $\operatorname{Re} Z \leqq 0$.

Remark. To consider $\alpha$ complex, one must pass to the obvious complex version of $\mathscr{H}$. We assume that this is done whenever necessary.

Proof. That $G_{\alpha}$ is holomorphic in $\alpha$ follows from Theorem VII-1-14 of [4], by precisely the argument in example 1.15 which follows the proof of that theorem. The details are omitted.

That $G_{\alpha}$ has a non-empty resolvent set follows from the observation that for $\operatorname{Re} \alpha \geqq 0, G_{\alpha}$ is maximal dissipative and for $\operatorname{Re} \alpha \leqq 0,-G_{\alpha}$ is maximal dissipative. All the $G_{\alpha}$ are restrictions of order one $\left(\operatorname{dim} D(T) / D\left(G_{\alpha}\right)=1\right)$ of the operator $T$ defined by

$$
\begin{gathered}
D(T)=\stackrel{0}{H_{1}}[0, \pi] \oplus\left(\begin{array}{l}
0 \\
H_{1}
\end{array}[0, \pi] \cap H_{2}[0, a] \cap H_{2}[a, \pi]\right), \\
T=\left(\begin{array}{ll}
0 & D^{2} \\
I & 0
\end{array}\right) \text { for } x \neq a .
\end{gathered}
$$

By Corollary III.6.34 of [4], it follows that $G_{\alpha}$ has compact resolvent either for all values of $\alpha$ or for none. For $\alpha=0, G_{0}$ is skewadjoint with a complete system of eigenvectors

$$
\text { ( } \pm \text { in } \sin n x, \sin n x \text { ), } n=1,2, \ldots
$$

with eigenvalues $\pm i n$. Thus $G_{0}^{-1}$ is compact and $G_{\alpha}$ has compact resolvent for all $\alpha$.

To prove the simplicity of the eigenvalues, notice that the equation $G_{\alpha} U=i \lambda U$ for $U=(v, w)$ is equivalent to

$$
\begin{aligned}
D^{2} w & =i \lambda v, x \neq a, \\
v & =i \lambda w, \\
{[w] } & =0 \text { and } \alpha v=[D w] \text { at } x=a .
\end{aligned}
$$


Eliminating $v$ yields

$$
\begin{gathered}
D^{2} w+\lambda^{2} w=0 \text { for } x \neq a, \\
i \lambda \alpha w=[D w] \text { and }[w]=0 \text { at } x=a .
\end{gathered}
$$

Given two eigenvectors with the eigenvalue $i \lambda$, we may form a linear combination $(v, w)$, such that $w^{\prime}(0)=0$. Then since $w(0)=0$ the differential equation (3.1) implies $w \equiv 0$, on $[0 a]$. The transmission conditions (3.2) then show that $D w(a+)=(w a+)=0$, and equation (3.1) yields $w \equiv 0$ on $[a, \pi]$. Hence $w=0$ and therefore $v=i \lambda w=0$. Thus $(v, w)=0$. This shows that any two eigenvectors with the same eigenvalue are linearly dependent.

The analytic dependence on $\alpha$ is now a consequence of the fact that $G_{\alpha}$ is holomorphic in $\alpha$ ([4, thm. VII.1.7]).

The analyticity in $a$ is a little harder since the location of the transmission condition is changing. Fortunately, a standard method takes care of this. Let

$$
y_{a}(x)= \begin{cases}\frac{\pi}{2 a} x & \text { if } 0 \leqq x \leqq a \\ \frac{\pi}{2(\pi-a)}(x-\pi)+\pi & \text { if } a \leqq x \leqq \pi\end{cases}
$$

The map $x \rightarrow y_{a}(x)$ is a homeomorphism $[0, \pi]$ onto itself which takes $a$ to $\pi / 2$. Denote the inverse mapping by $x_{a}(y)$ and define maps

by

$$
S_{a}=\mathscr{H} \rightarrow \mathscr{H}
$$

$$
\left(S_{a} U\right)(y)=U\left(x_{a}(y)\right) \text {. }
$$

The mapping $S_{a}$ is invertible, and $\tilde{G}_{a} \equiv S_{a}^{-1} G_{\alpha} S_{a}$ is the operator given by the following procedure:

$$
\begin{aligned}
& D\left(\tilde{G}_{\alpha}\right)=\left\{(v, w) \in \stackrel{0}{H}_{1}[0, \pi] \oplus\left(\stackrel{0}{H_{1}}[0, \pi] \cap H_{2}[0, a] \cap H_{2}[a, \pi]\right) \mid\right. \\
& \text { at } \left.x=a, \alpha v=\frac{\pi}{2(\pi-a)} D w\left(\frac{\pi}{2}+\right)-\frac{\pi}{2 a} D w\left(\frac{\pi}{2}-\right)\right\} \\
& \tilde{G}_{\alpha}=\left\{\begin{array}{l}
\left(\begin{array}{l}
0\left(\frac{\pi}{2 a}\right)^{2} D^{2} \\
I \frac{0}{v}
\end{array}\right), 0 \leqq y<\frac{\pi}{2} \\
\left(\begin{array}{l}
0 \frac{\pi}{2(a-\pi)} D^{2} \\
I \quad 0
\end{array}\right), \frac{\pi}{2}<y<\pi .
\end{array}\right.
\end{aligned}
$$

The operator $\tilde{G}_{x}$ has coefficients depending analytically on $a$, and the transmission condition at $y=\pi / 2$ depends analytically on $a$. Thus the family $\tilde{G}_{x}$ is holomorphic in $a$ (in the sense of [4, chap. III, $\S 1$ ]); since the eigenvalues 
of $\tilde{G}_{\alpha}$ and $G_{\alpha}$ are the same, we have proved the desired analytic dependence on $a$.

For $\operatorname{Re} \alpha \geqq 0, G_{\alpha}$ is dissipative. Accordingly its spectrum is in the half plane $\operatorname{Re} Z \geqq 0$ and the proof of Theorem 2 is complete.

Theorem 3. If $a / \pi$ is irrational and $\alpha>0$, then the spectrum of $G_{\alpha}$ is contained in the open half plane $\operatorname{Re} Z<0$. All solutions of the transmission problem $U_{t}=$ $G_{\alpha} U$ decay to zero as $t \rightarrow+\infty$; more precisely, $s-\lim _{t \rightarrow+\infty} e^{t G_{\alpha}}=0$. However $G_{\alpha}$ has eigenvalues of arbitrarily small real part, whence $\left\|e^{t G_{\alpha}}\right\|=1$ for all $t \geqq 0$.

Proof. We analyse more closely the conditions (3.1), (3.2) that must be satisfied by solutions of $G U=i \lambda U$. In the proof of the simplicity of the eigenvalues, we showed that $w^{\prime}(0) \neq 0$ if $w \neq 0$. Thus the differential equation (3.1) and Dirichlet conditions at $x=0, \pi$ imply that up to a scalar multiple $w$ must be given by

$$
w=\left\{\begin{array}{cc}
\sin \lambda x, & 0 \leqq x \leqq a, \\
b \sin \lambda(x-\pi), & a \leqq x \leqq \pi
\end{array}\right.
$$

Since $[w]=0$ at $x=a$, we must have

$$
\sin \lambda a=b \sin \lambda(a-\pi),
$$

and the transmission condition $i \lambda \alpha w=[D w]$ at $x=a$ yields

$$
-i x \sin \lambda a=\cos \lambda a-b \cos \lambda(a-\pi) \text {. }
$$

Now, purely imaginary eigenvalues correspond to real values of $\lambda$ for which the right hand side of (3.5) is real, so we must have $\sin \lambda a=0$. From (3.4), it follows that either $\sin \lambda(a-\pi)=0$ or $b=0$. However if $b=0$, then the right hand side of (3.5) is $\cos \lambda a= \pm 1 \neq 0$, so (3.5) cannot hold. Thus $\sin \lambda a=\sin \lambda(a-\pi)$ $=0$. Hence $\lambda a=n \pi$ and $\lambda(a-\pi)=m \pi$ for integers $n, m \neq 0$ (for $\lambda=0$, (3.3) yields $w \equiv 0$ ), so

$$
\frac{a}{\pi}=\left(\frac{1}{m}-\frac{1}{n}\right)^{-1} \frac{1}{m}
$$

a rational number, Thus, since $\frac{a}{\pi}$ is irrational, the assertion about the spectrum of $G_{\alpha}$ is proved.

The decay properties now follow by applying the next result, which is implicit in the work in Section 9 of [5].

Abstract Decay Theorem. If $A$ is a maximal dissipative operator on a Hilbert space $\mathscr{H}$ such that

1. A has no purely imaginary eigenvalues, and

2. A has compact resolvent,

then

$$
s-\lim _{t \rightarrow+\infty} e^{t A}=0
$$


Taking $A=G_{x}$, we obtain the required decay condition.

Next we show that $G_{a}$ has eigenvalues arbitrarily close to the imaginary axis. For $\lambda$ not real it is clear that $\sin \lambda a \cdot \sin \lambda(a-\pi) \neq 0$. Hence we may divide (3.5) by this product to obtain

$$
i x=\cot \lambda(a-\pi)-\cot \lambda a .
$$

Since $a / \pi$ is irrational, we may choose ([3, Theorem 36]) fractions $p / q$ (in lowest terms) with $q$ arbitrarily large such that

$$
\left|\frac{p}{q}-\frac{a}{\pi}\right|<\frac{1}{q^{2}} \text {. }
$$

The spectrum of $G_{0}$ consists of the numbers \pm in $n=1,2, \ldots$, with corresponding eigenfunctions ( \pm in $\sin n x, \sin n x$ ). Consider the eigenvalue $i \lambda(\alpha)$, which starts at $\alpha=0$ from the point $i q$ (that is $\lambda(0)=q$ ). We claim that $\lambda(\alpha)$ remains close to $q$ even for rather large $\alpha$.

To make this precise, choose $p / q$ satisfying (3.7) and let $\alpha_{c}>0$ be the smallest value of $\alpha$ such that $|\lambda(\alpha)-q|=1 / q$. Let

and

$$
E_{1}=q a-p \pi \quad\left(\text { so } 0<\left|E_{1}\right|<1 / q\right)
$$

$$
E_{2}=\lambda\left(\alpha_{c}\right)-q \quad \text { (so }\left|E_{2}\right|=1 / q, \operatorname{Im} E_{2}>0 \text { ). }
$$

If $\equiv$ denotes equality modulo an integer multiple of $\pi$, we have

$$
\begin{aligned}
& \lambda\left(\alpha_{c}\right) a=q a+E_{2} a \equiv E_{1}+E_{2} a, \\
& \lambda\left(\alpha_{c}\right)(a-\pi) \equiv E_{1}+E_{2} a-E_{2} \pi .
\end{aligned}
$$

The cotangent function is periodic of period $\pi$; thus (3.6) implies that

$$
i x_{c}=\cot \left(E_{1}+E_{2} a-E_{2} \pi\right)-\cot \left(E_{1}+E_{2} a\right) .
$$

Since the singular part of the Laurent expansion of cot $Z$ about $Z=0$ is $1 / Z$, we conclude that there is a positive constant $C$ such that, for $\left|Z_{1}\right|<1,\left|Z_{2}\right|<1$,

$$
\left|\cot Z_{1}-\cot Z_{2}\right| \geqq\left|\frac{1}{Z_{1}}-\frac{1}{Z_{2}}\right|-C .
$$

Using this estimate in (3.8) yields

$$
\left|\alpha_{c}\right| \geqq \frac{\left|E_{2} \pi\right|}{\left|E_{1}+(a-\pi) E_{2}\right|\left|E_{1}+E_{2} a\right|}-C \geqq \frac{\pi}{(\pi+1)^{2}} q-C .
$$

Thus for $q$ sufficiently large, we have $\left|\alpha_{c}\right| \geqq q / 10$. Consequently if $\alpha<q / 10$, then $|\lambda(\alpha)-q|<1 / q$. For fixed $\alpha$ this shows that there are eigenvalues $i \lambda(\alpha)$ arbitrarily close to the imaginary axis, and the proof is complete.

Theorem 4. If $\alpha>0, q / \pi$ is rational, and $M$ is the closed linear span of the eigenvectors of $G_{\alpha}$ with purely imaginary eigenvalues, then:

1. $M$ is precisely the closed linear span of the eigenvectors of $G_{0}$ which vanish at a, and $G_{0}=G_{\alpha}$ on such eigenvectors; 
2. $e^{t G_{0}}=e^{t G_{\alpha}}$ on $M$; in particular $e^{t G_{\alpha}}$ is unitary on $M$;

3. $M^{\perp}$ is invariant under the semigroup $e^{t G_{\alpha}}$. Furthermore there exist positive constants $C_{1}$ and $C_{2}$ depending only on $\alpha$ and a such that

$$
\left\|e^{t G_{\alpha}} U\right\| \leqq C_{1} e^{-C_{2} t}\|U\|
$$

for all $U \in M^{\perp}$ and $t \geqq 0$.

Proof. Suppose that $G_{\alpha} U=i \lambda U$ with $\alpha>0$ and $\lambda \in \mathbb{R}$. Then $U=(v, w)$, $v=i \lambda w$, and $w$ is given by (3.3) up to a scalar multiple, where $b$ satisfies (3.4) and (3.5). For $\lambda=0$, (3.3) shows that $w \equiv 0$. For $\lambda \in \mathbb{R} \backslash 0$ the imaginary part of (3.5) yields $\sin \lambda \alpha=0$, which shows that $w(a)=0$. Thus $[D w](a)=0$ and $w$ satisfies $D^{2} w+\lambda^{2} w=0$ on the entire interval $[0, \pi]$. It follows that $U=(v, w) \in D\left(G_{0}\right), \quad G_{0} U=i \lambda U, \quad$ and $\quad v(a)=\frac{1}{\alpha}[D w]=0 . \quad$ Conversely, if $G_{0} U=i \lambda U$ and $U(a)=0$, then $U=(v, w) \in D\left(G_{\alpha}\right)$ and $G_{\alpha} U=i \lambda U$; note here that the transmission condition $\alpha v=[D w]$ at $x=a$ is automatically satisfied since both sides vanish. Thus (1) is proved.

That $e^{t G_{\alpha}}=e^{t G_{0}}$ on $M$ follows from the fact that the two semigroups agree on finite linear combinations of the eigenvectors of $G_{0}$ and that this is a dense subset of $M$. That $M^{\perp}$ is invariant under $e^{t G_{\alpha}}$ follows by applying the following simple lemma to $C=e^{t G_{\alpha}}$.

Lemma 1. If $C$ is a linear contraction from the Hilbert space $\mathscr{H}$ to itself and $M \subset \mathscr{H}$ is a closed invariant subspace of $\mathscr{H}$ such that $C=M \rightarrow M$ is unitary, then $M^{\perp}$ is invariant under $C$.

Proof. Suppose $m \in M$ and $n \in M^{\perp}$; then for all $\varepsilon \in \mathbb{R}$ we have

$$
\|C(m+\varepsilon n)\|^{2} \leqq\|m+\varepsilon n\|^{2} .
$$

Expanding both sides and using the relations $\|C m\|^{2}=\|m\|^{2}$ and $(m, n)=0$, we get

$$
2 \varepsilon(C m, C n)+\varepsilon^{2}\|C n\|^{2} \leqq \varepsilon^{2}\|n\|^{2} .
$$

Thus $(C m, C n)=0$ for all $m \in M$. Since $C$ maps $M$ onto $M$, we have $C n \in M^{\perp}$. This proves the lemma.

Now $\left.e^{t G_{\alpha}}\right|_{M \perp}$ is a semigroup of contractions whose generator has no purely imaginary eigenvalues. Thus the abstract decay theorem shows that

$$
s-\left.\lim _{t \rightarrow \infty} e^{t G_{\alpha}}\right|_{M^{\perp}}=0 .
$$

The exponential decay asserted in (3) lies deeper. The idea of our demonstration is that for $a / \pi=p / q$ with $p$ and $q$ relatively prime integers we can find a simple and explicit formula for $e^{q^{-1} G_{\alpha}}$. Let $h=1 / q$ and suppose that the underlying space $\mathscr{H}$ is the complex Hilbert space $L^{2} \oplus \stackrel{0}{H}^{1}$. The form of the explicit solution is described in the following lemma. In the proof, the mappings $A$ and $D$ are described explicitly. 
Lemma 2. Let $h=1 / q$ be defined as above, and let $\alpha \geqq 0$. Then there are Hilbert spaces $\mathscr{K}$ and $\mathscr{E}$ with $\operatorname{dim} \mathscr{E}=4 q-1$, and a unitary map

such that

$$
\Lambda: \mathscr{H} \rightarrow \mathscr{K} \oplus L^{2}\left(\left(0, \frac{h}{2}\right) ; \mathscr{E}\right)
$$

1. $\mathscr{K}$ and $L^{2}\left(\left(0, \frac{h}{2}\right) ; \mathscr{E}\right)$ are invariant under $\Lambda e^{h G_{\alpha}} \Lambda^{-1}$;

2. $\Lambda e^{h G_{\alpha}} \Lambda^{-1} \uparrow \mathscr{K}=I d_{\mathscr{K}}$; 3. There is a $D \in \operatorname{Hom}(\mathscr{E})$ such that $\Lambda e^{h G_{\alpha}} \Lambda^{-1} \uparrow L^{2}\left(\left(0, \frac{h}{2}\right) ; \mathscr{E}\right)$ is multipli-
cation by $D$; that is,

$$
\left(\Lambda e^{h G_{\alpha}} \Lambda^{-1} V\right)(x)=D V(x) \text { for all } V \in L^{2}\left(\left(0, \frac{h}{2}\right) ; \mathscr{E}\right) \text {. }
$$

Before proving the lemma, we complete the proof of Theorem 4. Since $A e^{h G_{\alpha} \Lambda^{-1}}$ is a contraction, the same must be true of $D$. Write $\mathscr{E}=\mathscr{E}_{0} \oplus_{\perp} \mathscr{E}_{1}$, where $\mathscr{E}_{0}$ is the span of eigenvectors of $D$ with eigenvalues of modulus one. Then $D$ is unitary on $\mathscr{E}_{0}$, and there are constants $c>0$ and $\varrho \in(0,1)$ with

$$
\left\|D^{n} \uparrow \mathscr{E}_{1}\right\| \leqq C \varrho^{n}, n=0,1,2, \ldots .
$$

Corresponding to the decomposition of $\mathscr{E}$, we have a canonical decomposition

$$
L^{2}\left(\left(0, \frac{h}{2}\right) ; \mathscr{E}\right) \cong L^{2}\left(\left(0, \frac{h}{2}\right) ; \mathscr{E}_{0}\right) \oplus_{\perp} L^{2}\left(\left(0, \frac{h}{2}\right) ; \mathscr{E}_{1}\right)
$$

Define $M_{0} \subset \mathscr{H}$ by

$$
\Lambda M_{0} \equiv \mathscr{K} \oplus L^{2}\left(\left(0, \frac{h}{2}\right) ; \mathscr{E}_{0}\right) .
$$

Then $M_{0}$ has the following properties:

$$
\begin{aligned}
& e^{h G_{\alpha}}: M_{0} \rightarrow M_{0} \text { is unitary, } \\
& e^{h G_{\alpha}}: M_{0}^{\perp} \rightarrow M_{0}^{\perp},
\end{aligned}
$$

and

$$
\left\|e^{n h G_{\alpha}} \uparrow M_{0}^{\perp}\right\| \leqq C \varrho^{n}, n=0,1,2, \ldots .
$$

As a consequence, we must have $M_{0}=M$. Hence (3.9) yields part (3) of Theorem 4.

We return now to the proof of Lemma 2. Introduce the characteristic coordinates

$$
\xi=\frac{1}{\sqrt{2}}(t+x), \quad \eta=\frac{1}{\sqrt{2}}(t-x)
$$


and the characteristic derivatives

$$
\begin{aligned}
& u_{\xi}=\frac{\partial u}{\partial \xi}=\frac{1}{\sqrt{2}}\left(\frac{\partial u}{\partial t}+\frac{\partial u}{\partial x}\right), \\
& u_{\eta}=\frac{\partial u}{\partial \eta}=\frac{1}{\sqrt{2}}\left(\frac{\partial u}{\partial t}+\frac{\partial u}{\partial x}\right) .
\end{aligned}
$$

The wave equation (2.6) yields

$$
\frac{\partial^{2} u}{\partial \xi \partial \eta}=0 \text { for } x \neq a .
$$

Equivalently, $u_{\xi}$ is constant on the characteristics of speed -1 , and $u_{\eta}$ is constant on the characteristics of speed +1 .

The operator $A$ will be the product of unitary maps, the first one being the map which passes from the variables $\left(u_{t}, u\right)$ to the variable $\left(u_{\xi}, u_{\eta}\right)$. More precisely, we define $\Lambda_{1}: \mathscr{H} \rightarrow \mathscr{H}_{1}$ by

$$
\begin{gathered}
\mathscr{H}_{1} \equiv\left\{(v, w) \in L^{2}(0, \pi)^{2}: \int_{0}^{\pi}(v-w) d x=0\right\}, \\
\Lambda_{1}(\varphi, \psi)=\left(\varphi+\frac{\partial \psi}{\partial x}, \varphi-\frac{\partial \psi}{\partial x}\right) .
\end{gathered}
$$

Hence if

then

$$
\left(u_{t}(t), u(t)\right)=e^{t G_{\alpha}}(\varphi, \psi)
$$

$$
\Lambda_{1}\left(u_{t}(t), u(t)\right)=\left(u_{\xi}(t), u_{\eta}(t)\right)
$$

The inverse of $A_{1}$ is given by

$$
A_{1}^{-1}(v, w)=\left(\frac{1}{\sqrt{2}}(v+w), \frac{1}{\sqrt{2}} \int_{0}^{x}(v-w)(s) d s\right) .
$$

The condition $\int_{0}^{\pi}(v-w) d x=0$ in the definition of $\mathscr{H}_{1}$ reflects the fact that $u \in \stackrel{0}{H}_{1}$. The fact that $\mathscr{H}_{1}$ is not quite all of $L^{2}(0, \pi)^{2}$ will cause some small problems later on. The operator $A_{1} e^{h G_{x}} A_{1}^{-1}$ gives the operator "evolution by $h$ units of time in the $\left(u_{\xi}, u_{\eta}\right)$ variables". Because of $(3.10)$, the evolution of $\left(u_{\xi}, u_{\eta}\right)$ is particularly simple. To study this evolution, we decompose the interval $(0, \pi)$ into $q$ intervals of length $h$, the $j^{\text {th }}$ interval being

$$
((j-1) h, h), j=1,2, \ldots, q .
$$

We define $u_{\xi}^{j}, u_{\eta}^{j}$ to be the restriction of $u_{\xi}, u_{\eta}$ to the $j^{\text {th }}$ interval, translated to the reference position $(0, h)$, that is

$$
\begin{gathered}
u_{\xi}^{j}(t) \in L^{2}(0, h), \quad u_{\eta}^{j}(t) \in L^{2}(0, h), \\
u_{\xi}^{j}(t, x) \equiv u_{\xi}(t,(j-1) h+x), \quad 0<x<h, \\
u_{\eta}^{j}(t, x) \equiv u_{\eta}(t,(j-1) h+x), \quad 0<x<h .
\end{gathered}
$$


Equation (3.10) yields

$$
\begin{aligned}
& u_{\xi}^{j}(t+h)=u_{\xi}^{j+1}(t), \quad j=1,2, \ldots, p-1, p+1, \ldots, q-1, \\
& \text { (3.13) })_{\eta} \quad u_{\eta}^{j}(t+h)=u_{\eta}^{j-1}(t), \quad j=2,3, \ldots, p, p+2, \ldots, q \text {. }
\end{aligned}
$$

To complete the description of the evolution, we must give rules for determining

$$
u_{\eta}^{1}(t+h), u_{\xi}^{q}(t+h), u_{\xi}^{p}(t+h), \text { and } u_{\eta}^{p+1}(t+h),
$$

from $u_{\xi}(t)$ and $u_{\eta}(t)$. The first two of these are determined with the aid of the boundary condition

One finds

$$
u_{\xi}+u_{\eta}=0 \text { at } x=0, \pi
$$

$$
\begin{aligned}
& u_{\eta}^{1}(t+h)=-R u_{\xi}^{1}(t), \\
& u_{\xi}^{q}(t+h)=-R u_{\eta}^{q}(t),
\end{aligned}
$$

where $R=L^{2}(0, h) \rightarrow L^{2}(0, h)$ denotes reflection about $x=h / 2$, that is

$$
(R \varphi)(x)=\varphi(h-x), \quad 0<x<h .
$$

The transmission condition (2.7) provides the values of $u_{\xi}^{p}(t+h)$ and $u_{\eta}^{p+1}(t+h)$. Let

$$
\begin{array}{ll}
u_{\xi}^{-}(a, t)=\lim _{x \ngtr a} u_{\xi}(x, t), & u_{\xi}^{+}(a, t)=\lim _{x \searrow a} u_{\xi}(x, t), \\
u_{\eta}^{-}(a, t)=\lim _{x \ngtr a} u_{\eta}(x, t), & u_{\eta}^{+}(a, t)=\lim _{x \searrow a} u_{\eta}(x, t) .
\end{array}
$$

These quantities exist provided $\left(u_{t}(0), u(0)\right) \in \mathscr{D}\left(G_{a}\right)$. In fact, the entire calculation of $A_{1} e^{h G_{\alpha}} \Lambda_{1}^{-1}$ should be considered for such $u$ and then extended by continuity to $u$ with $\left(u_{t}(0), u(0)\right) \in \mathscr{H}$. The wave equation (3.10) yields

$$
\begin{array}{ll}
u_{\xi}^{+}(t+s, a)=u_{\xi}(t, a+s), & 0<s<h, \\
u_{\eta}^{-}(t+s, a)=u_{\eta}(t, a-s), & 0<s<h .
\end{array}
$$

In terms of the $u_{\xi}, u_{\eta}$ variables, the transmission conditions (2.7) become

$$
\begin{gathered}
u_{\xi}^{+}+u_{\eta}^{+}=u_{\xi}^{-}+u_{\eta}^{-}, \quad\left(\left[u_{t}\right]=0\right), \\
\left(u_{\xi}^{+}-u_{\eta}^{+}\right)-\left(u_{\xi}^{-}-u_{\eta}^{-}\right)=\alpha\left(u_{\xi}^{+}+u_{\eta}^{+}\right), \quad\left(\left[u_{x}\right]=\alpha u_{t}\right) .
\end{gathered}
$$

Solving these equations for $u_{\xi}^{-}$and $u_{\eta}^{+}$yields, for $\alpha \neq-2$,

$$
u_{\xi}^{-}=\frac{2}{2+\alpha} u_{\xi}^{+}-\frac{\alpha}{2+\alpha} u_{\eta}^{-}, \quad u_{\eta}^{+}=\frac{-\alpha}{2+\alpha} u_{\xi}^{+}+\frac{2}{2+\alpha} u_{\eta}^{-} .
$$

Using the previous expressions for $u_{\xi}^{+}$and $u_{\eta}^{-}$we find

$$
\begin{gathered}
u_{\xi}^{p}(t+h)=\frac{2}{2+\alpha} u_{\xi}^{p+1}(t)-\frac{\alpha}{2+\alpha} R u_{\eta}^{p}, \\
u_{\eta}^{p+1}(t+h)=\frac{-\alpha}{2+\alpha} R u_{\xi}^{p+1}(t)+\frac{2}{2+\alpha} u_{\eta}^{p}(t) .
\end{gathered}
$$


The formulas (3.13)-(3.15) give a simple expression for the time evolution of $\left(u_{\xi}, u_{\eta}\right)$. The reflections $R$ can be removed from these formulas by splitting

$$
U(t) \equiv\left(u_{\xi}^{1}, u_{\eta}^{1}, u_{\xi}^{2}, u_{\eta}^{2}, \ldots, u_{\xi}^{q}, u_{\eta}^{q}\right) \in L^{2}\left((0, h): \mathbb{C}^{2 q}\right)
$$

into its even and odd parts,

$$
\begin{aligned}
U & =U_{\text {even }}+U_{\text {odd }} \\
\left(U_{\text {even }}(t)\right)(x) & =\frac{1}{2}[U(t)(x)+U(t)(h-x)], \\
\left(U_{\text {odd }}(t)\right)(x) & =\frac{1}{2}[U(t)(x)-U(t)(h-x)] .
\end{aligned}
$$

From formulas (3.13)-(3.15) we see that the even and odd parts are preserved by the evolution, that is

$$
\left(\Lambda_{1} e^{h G_{\alpha}} \Lambda_{1}^{-1} U\right)_{\text {even }}=A_{1} e^{h G_{\alpha}} \Lambda_{1}^{-1}\left(U_{\text {even }}\right)
$$

with a similar formula with even replaced by odd.

Furthermore, on the even (respectively odd) parts $R$ acts as multiplication by 1 (respectively -1 ). Thus, if we let $A_{2}$ be defined by

$$
\begin{gathered}
\Lambda_{2}: L^{2}\left((0, h) ; \mathbb{C}^{2 q}\right) \rightarrow L^{2}\left(\left(0, \frac{h}{2}\right) ; \mathbb{C}^{4 q}\right) \\
\Lambda_{2} U=\frac{1}{2}\left(U_{\text {even }}, U_{\text {odd }}\right) \uparrow\left(0, \frac{h}{2}\right),
\end{gathered}
$$

and let

then

$$
\mathscr{H}_{2} \equiv \Lambda_{2} \mathscr{H}_{1} \subset L^{2}\left(\left(0, \frac{h}{2}\right) ; \mathbb{C}^{4 q}\right)
$$

$$
\begin{gathered}
\Lambda_{2}=\mathscr{H}_{1} \rightarrow \mathscr{H}_{2} \text { is unitary } \\
\Lambda_{2} \Lambda_{1} e^{h G_{\alpha}}\left(\Lambda_{2} \Lambda_{1}\right)^{-1}: \mathscr{H}_{1} \rightarrow \mathscr{H}_{2}
\end{gathered}
$$

and

(3.16) $A_{2} \Lambda_{1} e^{h G_{x}}\left(A_{2} A_{1}\right)^{-1}=$ multiplication by a $D_{1} \subset \operatorname{Hom}\left(\mathbb{C}^{4 q}\right)$.

From the definitions of $\mathscr{H}_{1}$ and $A_{2}$ we find that, for

$$
\begin{gathered}
\zeta \equiv(\underbrace{1,-1,1,-1, \ldots, 1,-1}_{2 q \text { entries }}, \underbrace{0,0, \ldots, 0)}_{2 q \text { entries }} \in \mathbb{C}^{4 q}, \\
\mathscr{H}_{2}=\left\{\phi \in L^{2}\left(\left(0, \frac{h}{2}\right) ; \mathbb{C}^{4 q}\right): \int_{0}^{h / 2}<\phi(x), \zeta>d x=0\right\} .
\end{gathered}
$$


If $\mathscr{H}_{2}$ were all of $L^{2}\left(\left(0, \frac{h}{2}\right) ; \mathbb{C}^{4 q}\right)$ the proof would be finished. To eliminate the "orthogonal to $\zeta$ " condition, we write

where

$$
\mathbb{C}^{4 q}=\mathbb{C} \zeta \oplus_{\perp} \mathscr{E}
$$

$$
\mathscr{E} \equiv\left\{\eta \in \mathbb{C}^{4 q}:\langle\eta, \zeta\rangle=0\right\}
$$

Corresponding to this decomposition, there exists a canonical decomposition

$$
\begin{aligned}
& L^{2}\left(\left(0, \frac{h}{2}\right) ; \mathbb{C}^{4 q}\right) \approx L^{2}\left(\left(0, \frac{h}{2}\right) ; \mathbb{C} \zeta\right) \oplus_{\perp} L^{2}\left(\left(0, \frac{h}{2}\right) ; \mathscr{E}\right) \\
& \mathscr{H}_{2} \approx \mathscr{K} \oplus_{\perp} L\left(\left(0, \frac{h}{2}\right) ; \mathscr{E}\right),
\end{aligned}
$$

where

$$
\mathscr{K} \equiv\left\{\psi \in L^{2}\left(\left(0, \frac{h}{2}\right) ; \mathbb{C} \zeta\right): \int_{0}^{h / 2}\langle\psi(x), \zeta\rangle d x=0\right\} .
$$

To finish the proof, we need two simple properties of the matrix $D_{1}$, namely

$$
D_{1} \zeta=\zeta
$$

and

$$
D_{1}^{*} \zeta=\zeta
$$

where $D_{1}^{*}$ is the adjoint of $D_{1}$. The first of these properties is equivalent to the easily verified fact that the evolution defined by (3.13)-(3.15) leaves invariant the function defined by

$$
u_{\xi}(t, x)=-u_{\eta}(t, x)=1, \quad 0<x<\pi .
$$

The second property follows from the first. Indeed (3.16) and (3.18), show that

$$
\Lambda_{2} \Lambda_{1} e^{h G_{\alpha}}\left(\Lambda_{2} \Lambda_{1}\right)^{-1} \uparrow \mathscr{K}=\operatorname{Id}_{\mathscr{K}} .
$$

Since $\alpha \geqq 0, A_{2} \Lambda_{1} e^{h G_{\alpha}}\left(\Lambda_{2} \Lambda_{1}\right)^{-1}$ is a contraction; hence Lemma 1 implies that $\mathscr{K}^{\perp}=L^{2}\left(\left(0, \frac{h}{2}\right) ; \mathscr{E}\right)$ is invariant. Thus we may form the adjoint of equation (3.19), to obtain

$$
\left[\Lambda_{2} \Lambda_{1} e^{h G_{\alpha}}\left(A_{2} \Lambda_{1}\right)^{-1}\right]^{*} \uparrow \mathscr{K}=\operatorname{Id}_{\mathscr{K}}
$$

because of (3.16) this is equivalent to the identity $(3.18)_{2}$.

The condition $D_{1}^{*} \zeta=\zeta$ implies $D_{1}(\mathscr{E}) \subset \mathscr{E}$. Consequently we may define $D \in \operatorname{Hom}(\mathscr{E})$ by $D \equiv D_{1} \uparrow \mathscr{E}$. If $A_{3}: \mathscr{H}_{2} \rightarrow \mathscr{K} \oplus_{\perp} L^{2}$ is the isomorphism in (3.17), then (3.16), (3.17) and (3.19) show that, with

$$
A=\Lambda_{3} \Lambda_{2} \Lambda_{1} \text {, }
$$

$\mathscr{K}$ and $D$ have the properties required in Lemma 2. 
Theorem 4 shows that if $\frac{a}{\pi}$ is rational, the spectrum of $G_{\alpha} \uparrow M^{\perp}$ must lie in a half plane $\operatorname{Re} Z \leqq-C_{2}$, while the spectrum of $G_{\alpha} \uparrow M$ is on the imaginary axis.

In contrast, Theorem 3 shows that if $\frac{a}{\pi}$ is irrational, the spectrum of $G_{\alpha}$ lies in the half plane $\operatorname{Re} Z<0$, but has points arbitrarily close to the imaginary axis.

These results all involve bounds on the spectrum, from the right. A complementary result, which is much more elementary is the following.

Theorem 5. For $a \in(0, \pi)$ fixed and $\alpha \neq-2$, the spectrum of $G_{\alpha}$ is contained in a strip $\operatorname{Re} Z \geqq C(\alpha)$, where the function $C(\alpha)$ is bounded on compact subsets of $\mathbb{C} \backslash\{-2\}$.

Proof. We need only consider eigenvalues $i \lambda$ with $\lambda \notin \mathbb{R}$. For these, equation (3.6) holds and the theorem follows from the observation that $\cot Z \rightarrow \pm i$ as $\operatorname{Im} Z \rightarrow \pm \infty$, the convergence being uniform in $\operatorname{Re} Z$.

The exceptional value $\alpha=-2$ occurs in another (not unrelated) context. For $\alpha \neq-2, G_{\alpha}$ is the generator of a one parameter group on $\mathscr{H}$, while for $\alpha=-2$, it only generates a semigroup. It is interesting to note that the higher dimensional analogues of our transmission problem, for example friction localized on a curvilinear subset of a membrane, are never reversible, that is if $\operatorname{Re} \alpha \neq 0$ one gets a semigroup and not a group. The proofs of these facts are omitted.

In case $a=\frac{\pi}{2}, e^{\pi G_{x}}$ can be computed without great effort. Though somewhat special, this result will play a role in our discussion of the significance of the model.

Theorem 6. If $a=\frac{\pi}{2}$ and $\alpha \neq-2$, then $e^{\pi G_{\alpha}} \uparrow M^{\perp}=\frac{\alpha-2}{\alpha+2} \operatorname{Id}_{M \perp}$.

Proof. We calculate, in somewhat more detail, the explicit solution constructed in the proof of Theorem 4. First of all, we observe that $M$ consists simply of those functions $\varphi, \psi$ which are even with respect to reflection in $x=\frac{\pi}{2}$, while $M^{\perp}$ consists of those functions which are odd. Thus for time evolution, it suffices to consider functions $u$ for which $u_{t}$ is even and $u_{x}$ odd, that is,

and

$$
\left(u_{\xi}+u_{\eta}\right)(t, x)=\left(u_{\xi}+u_{\eta}\right)(t, x-x)
$$

$$
\left(u_{\xi}-u_{\eta}\right)(t, x)=-\left(u_{\xi}-u_{\eta}\right)(t, \pi-x) .
$$

These relations are equivalent to

and

$$
R\left(u_{\xi}^{1}+u_{\eta}^{1}\right)=u_{\xi}^{2}+u_{\eta}^{2}
$$

$$
-R\left(u_{\xi}^{1}-u_{\eta}^{1}\right)=u_{\xi}^{2}-u_{\eta}^{2},
$$


where $R$ and $u_{\xi}^{1}, u_{\xi}^{2}, \ldots$ occur in equation (3.11)-(3.14); while also

$$
u_{\xi}^{2}=R u_{\eta}^{1} \quad \text { and } \quad u_{\eta}^{2}=R u_{\xi}^{1} \text {. }
$$

With these equations in mind, we may write the time evolution in terms of $u_{\xi}^{1}, u_{\eta}^{1}$ only. Equation $(3.15)_{\xi}$ yields

$$
u_{\xi}^{1}\left(t+\frac{\pi}{2}\right)=\frac{2}{2+\alpha} R u_{\eta}^{1}-\frac{\alpha}{2+\alpha} R u_{\eta}^{1}=\frac{2-\alpha}{2+\alpha} R u_{\eta}^{1}(t),
$$

while equation $(3.14)_{\eta}$ gives

$$
u_{i}^{1}\left(t, \frac{\pi}{2}\right)=-R u_{\xi}^{1}(t)
$$

Iterating, we obtain

$$
\left(u_{\xi}^{1}(t+\pi), u_{\eta}^{1}(t+\pi)\right)=\frac{\alpha-2}{\alpha+2}\left(u_{\xi}^{1}(t), u_{\eta}^{1}(t)\right),
$$

which is the desired result.

\section{Discussion}

It is not a simple matter to decide whether equation (1.1) with a highly localized friction describes the primary mechanism for the production of harmonics, for it seems impossible to measure directly the effect of a musician's finger. We must therefore rely on qualitative predictions of the model. Fortunately, we have obtained many such in Sections 2 and 3. The principal results assert that, given an initial configuration (dictated, for example, by plucking a string), the motion governed by (1.1) is approximated by the limiting transmission problem (2.6)(2.8), provided the friction $b(x)$ is localized. This limiting problem has the following properties:

1. If $\frac{a}{\pi}$ is irrational, all solutions tend to zero.

2. If $\frac{a}{\pi}$ is rational, the modes which vanish at $a$ are unaffected by the friction, while those in the orthogonal complement decay exponentially.

These properties coincide with the observed fact that to play harmonics one places one's finger at a point with $\frac{a}{L}(L=$ length of the string) where $a$ is rational with a small denominator. For other placements, one only hears a short lived thud. When playing harmonics, a musician removes his finger from the string after a short time. In view of the fact that for a friction $b(x)$ spread over a finite interval all solutions tend to zero, this seems wise. Presumably what is happening is that the rate of decay is much slower for the modes vanishing at $a$ (a fact that is rigorously true in the limit $b(x) d x=x \delta(x-a)$ ); thus the musician leaves his finger in contact with the string long enough to damp the components in $M^{\perp}$, 
but not those in $M$. As this desription indicates, the playing of harmonics is a delicate matter, a fact that can easily be verified by anyone inexperienced in the art.

There is an additional sensitivity to the artistry of the player, clearly indicated in Theorem 6. The object in playing harmonics is to obtain as rapid decay as possible on $M^{\perp}$.

For $a=\frac{\pi}{2}$ we have

$$
e^{\tau G_{\alpha}} \uparrow M^{\perp}=\frac{\alpha-2}{\alpha+2} I .
$$

Clearly, the desirable value of $\alpha$ is $\alpha=2$. The optimal strategy is to apply a friction with $b(x) d x \approx 2 \delta\left(x-\frac{\pi}{2}\right)$ for approximately $\pi$ units of time. Too much friction $(\alpha \geqslant 2)$ or too little $(\alpha \approx 0)$ yields a very slow damping on $M^{\perp}$. We expect that similar phenomena occur for other rational values of $\frac{a}{\pi}$. The player must strive to achieve the correct "touch", which for $a=\frac{\pi}{2}$ corresponds to $b(x) d x \approx 2 \delta\left(x-\frac{\pi}{2}\right)$. The idea of correct touch leads to an intriguing mathematical problem.

Given $\frac{a}{\pi}=\frac{p}{q}$, find the value of $\alpha$ which, in some sense, gives the most rapid decay for $e^{t G_{\alpha}} \uparrow M^{\perp}$.

Theorem 6 shows that $\alpha=2$ is the answer when $a=\frac{\pi}{2}$. It is of interest to know how strongly $\alpha$ depends on $\frac{p}{q}$.

The descriptions above correspond to common experience with harmonics. This is not to say that we consider equation (1.1) an exact model. What we believe is that localized frictional damping is a reasonable candidate for the primary mechanism in the playing of harmonics. To support this idea, one must know that similar qualitative behavior occurs when other effects are included, since it is more than likely that the finger introduces effects other than friction. For example, one might expect that the finger exerts a spring force on the string with a strongly localized positive spring constant $k(x)$. The basic equation of motion is then

$$
u_{t t}+b(x) u_{t}=u_{x x}-k(x) u
$$

and the energy

$$
\int_{0}^{\pi}\left(u_{t}^{2}+u_{x}^{2}+k u^{2}\right) d x
$$

is a decreasing function of time. If, as above, we consider a sequence $b_{n}$ and $k_{n}$ becoming increasingly localized, that is $b_{n} \rightarrow \alpha \delta_{a}, k_{n} \rightarrow \beta \delta_{a}(\beta=0$ is allowed), 
then the solutions of the equation (4.1) will converge to solutions of the transmission problem

$$
\begin{gathered}
u_{t t}-u_{x x}=0, \quad x \neq a, \\
{\left[u_{t}\right]=0, \alpha u_{t}=\left[u_{x}\right]-\beta u \text { at } x=a,} \\
u=0 \text { at } x=0, \pi .
\end{gathered}
$$

An analysis like that given in Section 3 shows that this problem behaves qualitatively like (2.6)-(2.8) provided $\alpha>0$. For example, regardless of the value of $a$, the solutions yield a contraction semigroup on $\mathscr{H}$, if $\mathscr{H}$ is given the norm

$$
\|(v, w)\|^{2}=\int_{0}^{\pi}\left(v^{2}+(D w)^{2}\right) d x+\beta u^{2}(a) .
$$

In addition, if $\frac{a}{\pi}$ is irrational all solutions decay, while if $\frac{a}{\pi}$ is rational the motion on the space $M$ of Theorem 4 is the same as the free motion. Finally, if $M^{\perp}$ is the orthogonal complement in the scalar product induced by (4.2), then $M^{\perp}$ is invariant and solutions in $M^{\perp}$ decay. Since the proofs are similar to those already presented, the details are omitted. The point is that the qualitative behavior of our model is somewhat stable under perturbations.

There is a different and very natural candidate for the effect of the musician's finger. One might expect that the finger acts as an impenetrable obstacle which constrains the string to lie below the finger. More exactly, if the position of the obstable is given by the functional relation $y=\varphi(x)$, with a form such as that indicated in Figure 3, one is lead to the constraint

$$
u(t, x) \leqq \varphi(x) \text { for all } t, x .
$$

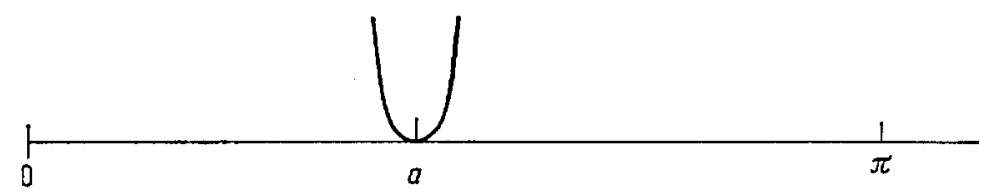

Fig. 4

Unfortunately, such problems are as yet poorly understood. On the other hand, the limiting case of an infinitely thin obstacle has been analysed by M. SCHATZMAN [9]. She has shown that for any $a \in(0, \pi)$ and any initial data $\left(u_{t}(0), u(0)\right) \in \mathscr{H}$ with $u(0, a) \leqq 0$, there is one and only one function $u$ such that

$$
\begin{gathered}
u(t, a) \leqq 0, \quad u_{t t}-u_{x x} \leqq 0, \\
\operatorname{supp}\left(u_{t t}-u_{x x}\right) \subset\{(t, a): u(t, a)=0\} .
\end{gathered}
$$

The first condition expresses the constraint exerted at the point $a$, the second and third say that the constraining force acts downward and is zero unless the string is in contact with the obstacle. 
A surprising consequence of these conditions is that the energy $\int_{0}^{x}\left(u_{t}^{2}(t, x)+\right.$ $\left.u_{x}^{2}(t, x)\right) d x$ is conserved. Explicit solution of several test problems shows that such a punctual constraint could provide a model for harmonics. In these examples one observes that the components of $u$ in $M$ are unaffected while the energy in $M^{\perp}$ is converted to energy in $M$ at high frequency. When $\frac{a}{\pi}$ is irrational, all energy moves into the high frequency range. Initially regular data lead to a superposition of a regular part and a jagged, high frequency part. Since the human ear has limited range, such an evolution could explain why one "hears" either a motion in $M$, or zero, depending on the rationality of $\frac{a}{\pi}$. The difference in the way the effect is achieved suggests an experimental test. One can observe in a laboratory the energy in high frequency modes (even those above the audible range). If energy seems to be pumped into these modes, this would favor the idea of a thin obstacle, while of the high frequency modes seem to be damped out, this would favor an explanation by a dissipative mechanism such as the friction we propose.

Note. This research was partially supported by the National Science Foundation under grants NSP GP 34260 and MCS 7802179.

\section{References}

1. R. Courant, Methods of Mathematical Physics, Vol. II, Interscience Publ., NewYork, 1962.

2. K. O. Friedrichs, Symmetric positive linear differential equations, Comm. Pure Appl. Math. 11 (1958), 333-418.

3. G. H. HARDY \& E. M. WRIGHT, Theory of Numbers, $4^{\text {th }}$ ed., Oxford U. Press, London, 1962.

4. T. Kato, Perturbation Theory for Linear Operators, Springer-Verlag, New-York, 1966.

5. P. D. LAX \& R. S. PhilliPS, Scattering theory for dissipative hyperbolic systems, J. Funct. Anal. 14 (1973), 172-235.

6. J. RAUCH \& F. MASSEY, Differentiability of solutions to hyperbolic initial-boundary value problems, Trans. A.M.S. 189 (1974), 303-318.

7. J. RAUCH \& M. TAYLOR, Exponential decay of solutions to hyperbolic equations in bounded domains, Indiana U. Math. J. 24 (1974), 79-86.

8. L. SARASON, On weak and strong solutions of boundary value problems, Comm. Pure Appl. Math. 15 (1962), 237-288.

9. M. Schatzman, Thèse de Doctorat, Université Pierre et Marie Curie, Paris, 1979.

Centre de Mathématiques Appliquées

Ecole Polytechnique

Palaiseau, France

Department of Mathematics

University of Michigan

Ann Arbor

Department of Mathematics

State University of New York

Stronybrook

(Received May 22, 1980) 